\title{
Acoustic sampling volume
}

\author{
Kenneth G. Foote \\ Institute of Marine Research, 5024 Bergen, Norway
}

(Received 5 April 1990; accepted for publication 10 April 1991)

\begin{abstract}
Knowledge of the acoustic sampling volume is necessary in many quantitative applications of acoustics. In general, the sampling volume is not merely a characteristic of the transmitting and receiving transducers, but also depends on the concentration and scattering properties of the target, the kind of signal processing performed on the echo, and the detection threshold. These dependences are stated explicitly in formulas for the sampling volume and a differential measure, the effective equivalent beam angle. Numerical examples are given for dispersed or dense concentrations of both point scatterers and directional fish scatterers. Application of theory to optical and other remote sensing techniques is mentioned.
\end{abstract}

PACS numbers: 43.30.Ft, 43.30.Gv, 43.30.Xm

\section{INTRODUCTION}

A number of practical uses of acoustics require knowledge of the sampling volume. In fluid-processing applications these include determinations of the concentration of monodispersed scatterers and the size distribution and concentration of polydispersed scatterers, e.g., of human red blood cells ${ }^{1}$ and other small particles, ${ }^{2-5}$ such as contaminants of industrial fluids. In oceanographic applications these include analogous determinations of scatterer concentration and size distribution, e.g., of bubbles, ${ }^{6}$ fish, ${ }^{7-9}$ plankton, ${ }^{10-13}$ and suspended sediment. ${ }^{14-17}$ Some of the cited applications involve bistatic sonar, others monostatic sonar, but the problem is the same: describing how large the particular sampling volume is.

In the case of high signal-to-noise ratio (SNR), as with acoustically powerful scatterers, the sampling volume may be identical to the available or accessible volume. However, in the case of low or marginal SNRs, the sampling volume will in general be less than the high-SNR volume. The reason is simply that some echoes from relatively weak scatterers, singly or aggregated, will lie below the detection threshold. This is not an academic situation either, for what technique in acoustics, or in science for that matter, is not at some time pushed to its limit: smaller, larger, weaker, stronger, thinner, denser, nearer, farther.

The problem of defining the acoustic sampling volume is especially timely in fisheries acoustics. Several years ago, when the Northeast Arctic cod (Gadus morhua) was surveyed during its annual migration to spawning grounds in Lofoten, the fish was observed at depths exceeding $400 \mathrm{~m}$, which is much deeper than usual. Since the echo sounding equipment begins to be severely limited with respect to the detection of single cod at this range, only a fraction of the stock was registered, as was later demonstrated by the catch. Other evidence for the importance of sampling-volume considerations for cod has been presented by Ona. ${ }^{18}$

Defining the sampling volume for fish registration is also particularly illustrative for the general problem because of the involved scattering regime. This is characterized by size-wavelength ratios of about 1-100, which are due to the use of ultrasonic survey frequencies in the approximate range $30-200 \mathrm{kHz}$. Consequently, a scatterer that gives a strong echo when in one orientation may give a very weak or unobservable echo when in another, despite being in the same position in the beam. Differences in backscattering cross sections due to ordinary changes in tilt angle will moreover be slight for small size-wavelength ratios and potentially large for big ratios, thus nearly spanning the range of effects intrinsic to the scatterer itself.

The dependence of the sampling volume on the backscattering characteristics of observed fish, in addition to transducer properties, has already been recognized. ${ }^{7,18-25}$ Dependence of the sampling volume on the minimum detectable signal level or so-called detection threshold has also been recognized. However, notwithstanding several different approaches to the problem, computations are scarce, and there is a distinct lack of concise or comprehensive expressions for the sampling volume or threshold effect.

A notable, overtly statistical approach to a different but related problem is that by Weimer and Ehrenberg. ${ }^{26}$ For a given threshold, the effect on a distribution of target strengths is described by an integral. This is evaluated numerically for a specific normal target strength distribution for each of several thresholds.

The present approach attempts to address both the particular fisheries acoustics application and the more general problem of defining the sampling volume. Following a description of the first-order role of the sampling volume in two methods of scatterer density determination, the theory of the sampling volume is developed. General issues concerned with integration are discussed. The volume associated with acoustic sampling by fish is computed through a derived differential measure, the effective equivalent beam angle, for a particular computational model. This is intended both to illustrate the method of numerical evaluation and to reveal some important dependences of the sampling volume on the underlying scatterer characteristics.

\section{THEORY}

Two common methods of determining scatterer concentration are those of echo counting and echo integration. ${ }^{27,28}$ In the case of sufficiently dispersed scatterers, the echo 
counting method may be used. According to this, the scatterer density $\rho$ is measured by the average number of echoes $\bar{N}$, obtained from the sampled volume $V_{s}$ per sounding,

$$
\rho=\bar{N} / V_{s} \text {. }
$$

The echo integration method may be applied in the general case of scatterers of arbitrary concentration. ${ }^{29}$ Accordingly, the column scattering coefficient $s_{a}$ is obtained by integrating the volume backscattering coefficient $s_{v}$ over an accessible range interval. The fundamental quantity $s_{v}$ relates the mean cumulative backscattering cross section $\bar{\sigma}$ per sounding to the sampled volume $V_{s},{ }^{12}$

$$
s_{v}=\bar{\sigma} /\left(4 \pi V_{s}\right),
$$

assuming, for the sake of simplicity only, negligible extinction. Usually $s_{v}$ is expressed as a function of range, or depth, by limiting $V_{s}$ by a succession of generally narrow range intervals. These two methods are well known in underwater acoustics, but possess exact analogs for the sampling of other media, whether by acoustical or optical means.

In the following, the theory of the sampling volume is first developed without reference to any particular method or application. It thus encompasses the bistatic case of separate transmitting and receiving transducers. The derived expression for the sampling volume is then specialized to the monostatic case of a single transducer or collocated transmitting and receiving transducers.

\section{A. Bistatic case}

In the general bistatic case, separate transducers are used for transmission and reception. The respective directional characteristics are contained in the one-way beam patterns $b_{T}$ and $b_{R}$. A received echo is registered if its strength exceeds a minimum signal level or threshold $t$. The received echo strength is expressed as the product of a gain or geometric factor $g$, product of transmit and receive beam patterns $b^{2}$, and bistatic, or differential, cross section $\sigma$.

For a nondirectional scatterer with constant $\sigma$, the sampling volume $V_{s}$ is a fraction of the total available or accessible volume $V_{0}$ :

$$
V_{s}=\int_{V_{0}} H\left(g b^{2} \sigma-t\right) d V .
$$

The integrand is a counting function: the Heaviside step function, $H(x)=0, \frac{1}{2}, 1$ as $x<0, x=0, x>0$, respectively. Thus, for echo strengths $g b^{2} \sigma$ exceeding $t$, the contribution is fully registered.

For directional scatterers, $\sigma$ varies with orientation. To account for this in $V_{s}$, the integration in Eq. (1) is also performed over the range of orientations determining the sampled values of $\sigma$ according to the cumulative distribution function $F$. Thus

$$
V_{s}=\iint H\left(g b^{2} \sigma-t\right) d F d V
$$

This is tantamount to Eq. (7) in Ref. 30, although with differences in nomenclature. For the case of constant $\sigma$, the integration over $d F$ yields unity and Eq. (1) results.

\section{B. Monostatic case}

The expression for $V_{s}$ in Eq. (2) is complete and unambiguous. However, its incorporation in echo counting and integration schemes, ${ }^{7,28}$ in their usual monostatic forms, requires adapting the equivalent beam angle $\psi_{0}$, which is defined entirely in terms of the transducer beam pattern, ${ }^{31}$

$$
\psi_{0}=\int b^{2} d \Omega
$$

Since this applies at a constant, far-field range, and $d V=r^{2} d r d \Omega$, the solid-angle analog to Eq. (2) is

$$
\iint H\left(g b^{2} \sigma-t\right) d F d \Omega,
$$

where $\sigma$ is the backscattering cross section. Comparing this with Eq. (3), it is clear that the effective equivalent beam angle is

$$
\psi=\iint b^{2} H\left(g b^{2} \sigma-t\right) d F d \Omega .
$$

This quantity can, in one sense, be regarded as a generalization of the equivalent beam angle defined in Eq. (3). However, its origin is in the concept of sampling volume, described in Eq. (2), and, when $\psi$ is multiplied by $r^{2} \Delta r$, the product is equal to the sampling volume within a spherical shell of infinitesimal thickness $\Delta r$.

The gain factor $g$ in the several equations is exemplified by two extreme, but not uncommon, monostatic situations of detection in the usual far field of the transducer: (1) for a single scatterer, $g=10^{-\alpha r / 5} r^{-4}$, where $\alpha$ is the coefficient of absorption given in decibels per meter and $r$ is the range in meters to the scatterer; and (2) for a layer of identical scatterers, $g=10^{-\alpha r / 5} r^{-2}$.

The detection threshold $t$ has the same units as the product $g b^{2} \sigma$. At the very threshold, detection occurs essentially on the acoustic axis, where $b=1$. The scatterer, if directional, is moreover in its most favorable aspect, where $\sigma=\sigma_{\max }$. Here, at the maximum detection range, $g$ is a minimum. Thus $t=g_{\min } \sigma_{\max }$. In the limit that $t$ vanishes, or the SNR becomes very large, $V_{s} \rightarrow V_{0}$ and $\psi \rightarrow \psi_{0}$.

\section{INTEGRATION ISSUES}

In both the general expression for $V_{s}$ in Eq. (2) and the associated differential measure for the monostatic case, $\psi$ in Eq. (4), the spatial integrals are shown without explicit limits, and the Heaviside step function $H$ appears in the integrand. The integration with respect to the volume element $d V$ in Eq. (2) is understood to be performed over the entire volume that is accessible between the range intervals of interest. Similarly, the integration with respect to the solid-angle element $d \Omega$ in $\mathrm{Eq}$. (4) is assumed to be performed over that accessible at the particular range of interest. The physically available space, either volume or solid angle, constitutes an upper bound to the respective integrals.

The Heaviside step function, or counting function, describes the details of the sampling process. If and only if the received echo strength $g b^{2} \sigma$ exceeds the detection threshold $t$ is the echo fully registered. In the case that $g b^{2} \sigma=t$, the echo is registered with one-half weight or count, corresponding to 
the statistical registration of such marginal echoes $50 \%$ of the time.

For generally directive scatterers with random orientations, echoes arising from the same scatterer at the same fixed position in space may or may not be registered. In a favorable orientation, for example, the scattering cross section $\sigma$ may be so large that $g b^{2} \sigma$ exceeds $t$ and the echo is registered. In an unfavorable orientation at the same position, $g b^{2} \sigma$ may be less than $t$, and the echo will not be registered. It is therefore not generally possible to remove the Heaviside step function from the integrand and impose precise limits on the volume or solid-angle integrals. That is, $V_{s}$ and $\psi$ are not sharply defined regions of space. Nonetheless, they are well-defined quantities, as described in the several integrals.

The discussed integrals, if analytically intractable, are, in fact, easy to compute by means of an algorithm. The physically available space is first partitioned into small cells. Each cell is then examined separately with respect to the received echo level relative to the detection threshold for the range of scatterer orientations specified by the distribution function $F$. The several continuous variables involved in this are discretized in the usual manner, as illustrated by the example in Sec. IV. Received echo levels are, respectively, counted or ignored as $g b^{2} \sigma$ exceeds or is less than $t$.

\section{COMPUTATIONAL MODEL FOR ACOUSTIC SAMPLING OF FISH}

An immediate application of theory is in the acoustic measurement of fish density. It is convenient to evaluate numerically the effective equivalent beam angle in Eq. (4). This is done through the following model.

Medium. This consists of seawater of salinity $35 \mathrm{ppt}$ and temperature $5^{\circ} \mathrm{C}$. The sound speed is thus $1470 \mathrm{~m} / \mathrm{s} .^{32}$ At 38 $\mathrm{kHz}$, therefore, the absorption coefficient $\alpha$ is $0.0106 \mathrm{~dB} /$ $\mathrm{m}^{33}$

Transducer. The transducer is assumed to be circular, with half-beamwidth of $4 \mathrm{deg}$ or full beamwidth between opposite -3 -dB levels of $8 \mathrm{deg}$. The beam pattern thus depends only on the polar angle $\theta$, and $b=\left[2 J_{1}(k a \sin \theta) /\right.$ $(k a \sin \theta)]^{2}$, where $k a=1.61 / \sin (\pi / 45) \doteq 23.1$. Performance of the integration in Eq. (3) yields the nominal equivalent beam angle $\psi_{0}=0.0108$ sr or $-19.66 \mathrm{~dB}$.

Fish backscattering cross section. The source of data consists of measurements by Nakken and Olsen ${ }^{34}$ of the tilt an-

TABLE I. Characteristics of four subsets of target strength functions for gadoids at $38 \mathrm{kHz}$, used in computations for Figs. 1-4. The minimum and maximum lengths $l_{\min }$ and $l_{\max }$ refer to criteria applied in selecting the subsets. The number of included functions is denoted $n_{s}$. The mean and standard deviation of lengths $l$ in each subset are shown. Units of length are centimeters.

\begin{tabular}{|c|c|c|c|c|c|}
\hline \multirow[b]{2}{*}{ Subset } & \multirow[b]{2}{*}{$l_{\min }$} & \multirow[b]{2}{*}{$l_{\max }$} & \multirow[b]{2}{*}{$n_{s}$} & \multicolumn{2}{|c|}{$\hat{l}$} \\
\hline & & & & mean & s.d. \\
\hline 1 & 5 & 15 & 27 & 10.7 & 2.3 \\
\hline 2 & 15 & 25 & 29 & 21.5 & 3.0 \\
\hline 3 & 35 & 45 & 29 & 39.0 & 3.1 \\
\hline 4 & 55 & 65 & 21 & 59.0 & 3.2 \\
\hline
\end{tabular}

gle dependence of the dorsal aspect target strength function, which are tabulated in Ref. 35. Of these, 171 apply to the gadoids cod (Gadus morhua), saithe (Pollachius virens), and pollack (Pollachius pollachius), at $38 \mathrm{kHz}$. Four subsets of these functions are selected to illustrate the effect of fish length, thence directionality of scattering pattern, on $\psi$. These are described in Table I. The backscattering cross section $\sigma$ of fish at tilt angle $\theta^{\prime}$ is derived from the target strength value TS $\left(\theta^{\prime}\right)$ according to the definition ${ }^{36}$ TS $=10 \log \sigma /$ $4 \pi$, but with use of SI units.

Fish behavior. This is characterized in the usual way by a normal probability density function of tilt angle $N\left(\overline{\theta^{\prime}}, s_{\theta^{\prime}}\right)$. Two sets of parameters are used: $\left(\overline{\theta^{\prime}}, s_{\theta^{\prime}}\right)=(0,5)$ and $(-4.4,16.2) \mathrm{deg}$. The empirical bases of the two sets are described in Refs. 37 and 38, respectively. The tilt angle distribution is assumed to be truncated at two standard deviations from the mean. Thus the probability density function $f$ in $d F=f d \theta^{\prime}$ is $f \doteq 0.95^{-1} \exp \left[-\left(\theta^{\prime}-\overline{\theta^{\prime}}\right)^{2} / 2 s_{\theta^{\prime}}^{2}\right]$.

\section{NUMERICAL METHOD}

The integration in Eq. (4) is effected in the following way. For the range $r$, less than the maximum detection range $r_{\max }$, the equation $g b^{2}=t$ is solved for $\theta$. Specifically, the equation

$$
2 J_{1}(k a \sin \theta) / k a \sin \theta=10^{-\alpha\left(r_{\max }-r\right) / 20}\left(r / r_{\max }\right)^{q}
$$

is solved numerically, where $q=1$ for a single point scatterer and $q=1 / 2$ for a scattering layer. The solution, denoted $\theta_{r}$, is then used to limit the $\theta$ integration in Eq. (4), for the target at $r$ cannot be detected anywhere outside the cone $\theta=\theta_{r}$.

Equation (4) is evaluated in the following discrete version:

$$
\begin{aligned}
\psi_{r}= & 2 \Delta \theta \Delta \phi \sum_{i=1}^{n_{i}} b^{2}\left(\theta_{i}\right) \sin \theta_{i} \\
& \times \sum_{j=1}^{n_{j}}\left\{\left[\sum_{k=1}^{n_{k}} H\left(b^{2}\left(\theta_{i}\right) \frac{\sigma\left(\chi_{i j k}\right)}{\sigma_{\max }}-\frac{g_{\min }}{g_{r}}\right) f^{\prime}\left(\theta_{k}^{\prime}\right)\right]\right. \\
& \left.\times\left(\sum_{k=1}^{n_{k}} f^{\prime}\left(\theta_{k}^{\prime}\right)\right)^{-1}\right\},
\end{aligned}
$$

where

$\Delta \theta=\theta_{r} / n_{i}, \quad \theta_{i}=(i-1 / 2) \Delta \theta, \quad \Delta \phi=\pi / n_{j}$,

$\phi_{j}=(j-1 / 2) \Delta \phi, \quad \Delta \theta^{\prime}=4 s_{\theta^{\prime}} / n_{k}$,

$\theta_{k}^{\prime}=\overline{\theta^{\prime}}-2 s_{\theta^{\prime}}+(k-1 / 2) \Delta \theta^{\prime}$,

$\chi_{i j k}=\pi / 2-\cos ^{-1}\left(\sin \theta_{i} \cos \phi_{j} \cos \theta_{k}^{\prime}-\cos \theta_{i} \sin \theta_{k}^{\prime}\right)$.

The subscript is attached to $\psi$ and $g$ to emphasize their applicability at range $r$. In the computations reported below, $n_{i}=20, n_{j}=6$, and $n_{k}=40$.

\section{RESULTS}

The effective equivalent beam angle $\psi$ is examined first for a single point scatterer and a layer of point scatterers. Equation (4), thence Eq. (5) also, is immediately simplified, for the scattering is independent of orientation; hence, the integration over $d F$ yields unity. Since $b$ only depends on 


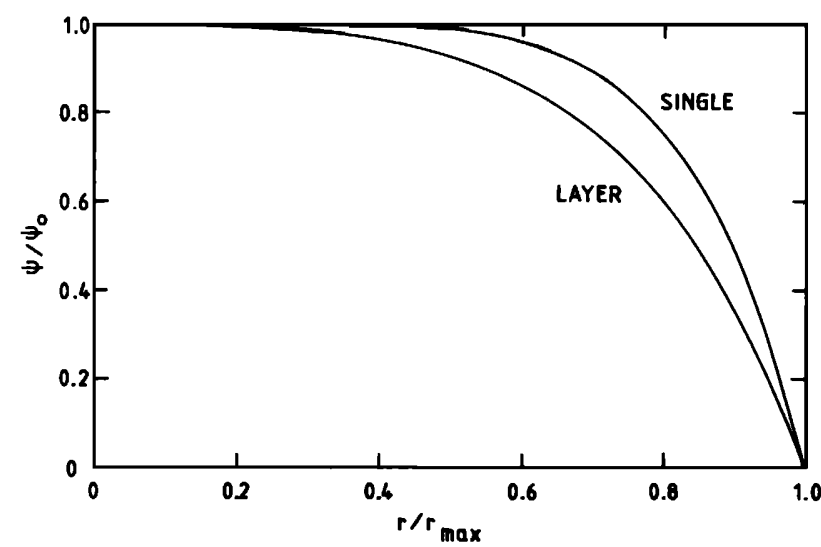

FIG. 1. Effective equivalent beam angle $\psi$ normalized to the nominal transducer value $\psi_{0}$ as a function of range $r$ relative to the maximum detectable range $r_{\max }=400 \mathrm{~m}$ for both a single point scatterer and a layer of identical point scatterers. Other parameters are specified in Sec. III.

$\theta$, integration over $\phi$ yields $2 \pi$. Given a maximum range of detectability, $\psi$ is reduced to the following:

$$
\psi_{r}=2 \pi \int b^{2}(\theta) H\left(b^{2}-\frac{g_{\min }}{g_{r}}\right) \sin \theta d \theta .
$$

This, or rather its discrete version, analogous to Eq. (5), is evaluated for $r_{\max }=400 \mathrm{~m}$. The results, after normalization to the ideal limit $\psi_{0}$, are presented in Fig. 1.

What is to be remarked on here, with force for the other computations too, is that an absolute comparison of the scattering strengths of the point scatterer and layer of point scatterers is not undertaken. Rather, each of two problems is examined, where each scatterer type has its detection threshold at $400 \mathrm{~m}$. Under ordinary conditions, without this constraint, if the point scatterers in the layer were identical with the single point scatterer, the detection thresholds would of course be different.

The effect of directionality in scattering by fish on $\psi$ is illustrated in Figs. 2-4 for the single-scatterer case, hence with $g=10^{-\alpha r / 5} r^{-4}$. Figure 2 applies to the tilt angle distribution $N(0,5)$; Fig. 3 applies to the distribution

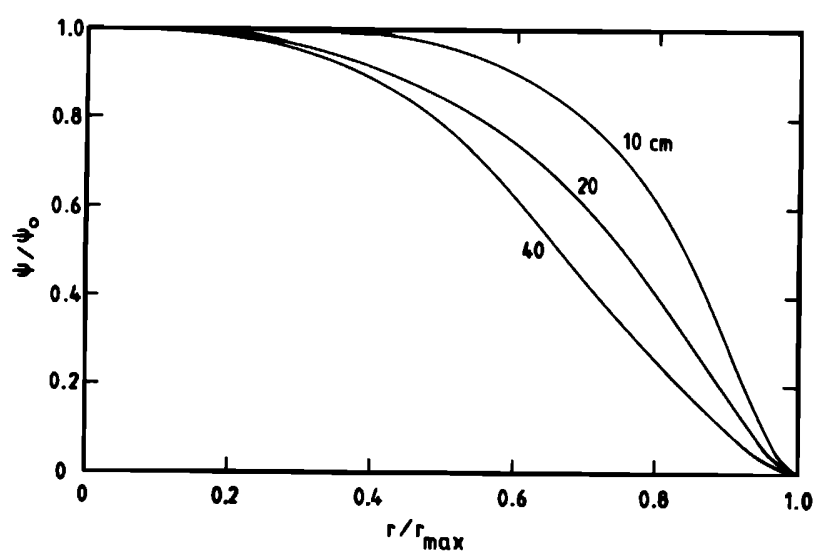

FIG. 2. Effective equivalent beam angle $\psi$, after normalization, versus range $r$ relative to $r_{\max }=400 \mathrm{~m}$ for gadoid target strength functions at $38 \mathrm{kHz}$, as described in subsets 1-3 in Table I, with respective nominal mean lengths 10,20 , and $40 \mathrm{~cm}$, assuming the tilt angle distribution $N(0,5) \mathrm{deg}$.

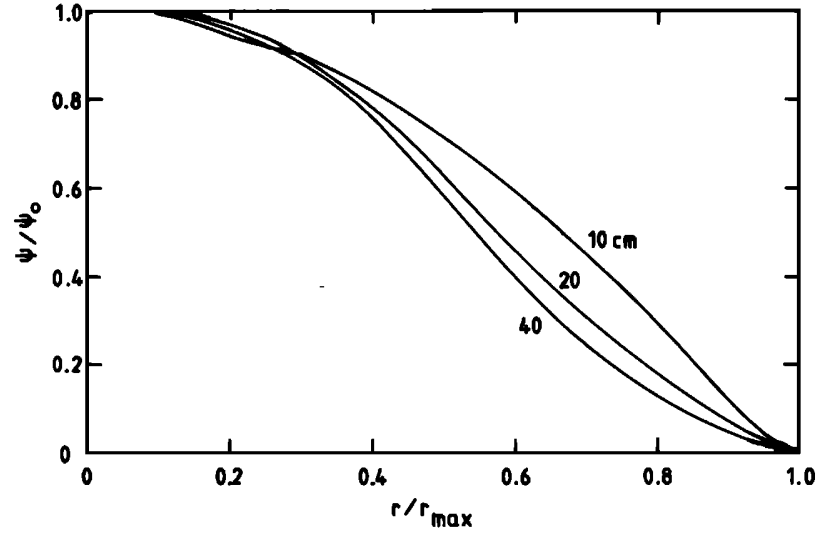

FIG. 3. Effective equivalent beam angle $\psi$, after normalization, versus range $r$ relative to $r_{\max }=400 \mathrm{~m}$, as in Fig. 2, but with the tilt angle distribution $N(-4.4,16.2)$ deg.

$N(-4.4,16.2)$. The effect of behavior on $\psi$ is shown directly for gadoids of nominal length $60 \mathrm{~cm}$ in Fig. 4 .

\section{DISCUSSION}

A number of systematic dependences expected from Eq. (4) are confirmed by the computations. To elucidate these more strongly, the dependence on the backscattering cross section $\sigma$ is essentially eliminated in the computations for Fig. 1 by consideration of identical point scatterers. For these, the value of the product $g b^{2}$, when compared with the threshold value $t$, is decisive for determining whether an echo strength lies above or below $t$, hence is or is not detected. Since the so-called gain or geometric factor $g$ decreases with increasing range, the maximum angle of detection, $\theta=\theta$, in the beam pattern $b$, also decreases with increasing $r$. This is evident in Fig. 1.

The curves in Figs. 1-4, which are ogives in Figs. 2-4, show the expected monotonic decrease in $\psi$ with increasing $r$. In addition, $\psi$ is seen to vanish at the maximum range $r_{\max }$ and to approach the nominal transducer value $\psi_{0}$ asymptotically as $r$ decreases.

Another systematic dependence seen in Fig. 1 is the effect of scatterer type, single or layer, on $\psi$. The mechanism

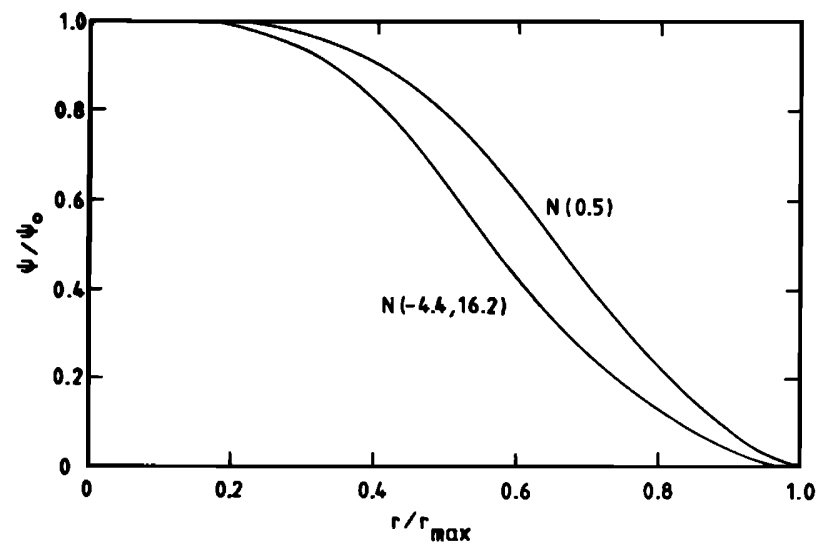

FIG. 4. Comparison of the normalized effective equivalent beam angle $\psi$ versus range $r$ relative to $r_{\max }=400 \mathrm{~m}$ for gadoid target strength functions, described in subset 4 of Table I, with nominal mean length $60 \mathrm{~cm}$, as computed for each of two tilt angle distributions. 
for this is the range dependence of $g$. For the same range, $g$ for the single point scatterer is less than $g$ for the layer of identical point scatterers, hence $\psi$ for the single-point scatterer exceeds that for the layer. If this result seems contrary in the context of overall backscattering strength, it must be remembered that the maximum detection range is assumed to be the same for the two scatterer types. This assumption is artificial for, all things being equal, the layer of identical scatterers would be detected at a greater range than the single scatterer would be. However, it was not felt necessary to illustrate this fact here.

Repetition of the computations in Fig. 1 for different values of $r_{\max }$ yields very similar results. For example, for a single point scatterer with $r_{\max }=500 \mathrm{~m}, \psi$ is within $1 \mathrm{~dB}$ of $\psi_{0}$ for ranges less than $390 \mathrm{~m}$, or $78 \%$ of $r_{\max }$, while with $r_{\max }=200 \mathrm{~m}, \psi$ departs from $\psi_{0}$ by $1 \mathrm{~dB}$ at $150 \mathrm{~m}$, or $75 \%$ of $r_{\max }$. The trend is indeed similar; the small difference is due to the absorption part of $g$, which does not scale with $r$ in the same way that the spreading part does.

Having established and shown how the effective equivalent beam angle $\psi$ varies for identical point scatterers, different single-fish scatterers are now considered. Comparison of the single-scatterer curve in Fig. 1 with any of the other curves in Figs. 2-4 shows that the effect of directionality in fish scattering is to decrease $\psi$ below that of the point scatterer. In addition, for the same orientation distribution, the larger the scatterer and more directional the scattering pattern, the smaller $\psi$ tends to be at the same $r$, assuming identical values for $r_{\max }$. This general trend clearly holds in Figs. 2 and 3, except for small values of $r / r_{\max }$ in Fig. 3, where the differences are not significant. The corresponding curves for $60-\mathrm{cm}$ fish in Fig. 4 also deviate somewhat from the trend, but again only to an insignificant degree. The discrepancies reflect variations in scattering properties, especially with respect to scatterer orientation, that are intrinsic to the scattering process, but which are not so strong as to upset the described general trend.

Behavior, as described by the orientation distribution, is also an important factor affecting $\psi$ in Eq. (4). For broad tilt angle distributions, such as that in Fig. 3, the chance of sensing lower values of backscattering cross section is much greater than for rather narrow distributions, such as that in Fig. 2, hence the general displacement of the two sets of curves. This is illustrated directly in Fig. 4, where the curve for $N(-4.4,16.2)$ lies significantly below that for $N(0,5)$ for the same set of fish target strength functions, subset number 4 in Table $I$.

As described in Sec. III, the computations presented in Figs. 1-4 are based on an ideal circular transducer with 4deg half-beamwidth, measured from the acoustic axis to - 3-dB level. Repetition of the computations for other narrow transducer beams, with half-beamwidths over the range 2.5-10 deg, shows no or only negligible differences with the present results.

The several functions described graphically can also be approximated. One successful function that has been used to model $\psi$ for cod target strength functions is the following: ${ }^{39}$

$$
\psi / \psi_{0}=\hat{\psi}_{1}+\hat{\psi}_{2},
$$

where

$$
\hat{\psi}_{1}=1-\left[1+(Q \Delta)^{2}\right]^{-1},
$$

$\Delta=r / r_{\max }-r_{\max } / r$, and $Q$ is a measure of the steepness of falloff of $\psi / \psi_{0}$. The function $\hat{\psi}_{2}$ is determined by fitting a Fourier series, such as finite cosine series, to the residual function

$$
\psi_{\text {res }}=\psi / \psi_{0}-\hat{\psi}_{1} \text {. }
$$

A fairly reasonable agreement can be obtained for a seventerm series, for example, but values near the threshold are most difficult to fit, both because of the smallness of the data sample and the sensitivity of $\psi$ near the threshold to the exact form of the target strength function.

While the model and computational example are directed to acoustic scattering, analogs exist in optical and other systems using irradiation and scattering for the remote sensing of scatterer concentration or size. Acknowledgment of the importance of the sampling volume is evident in the study of a forward-scattering system by Hirleman et al. ${ }^{40}$ Application to size and concentration measurements, by like forward scattering ${ }^{41}$ or by backscattering lidar or laser radar, ${ }^{42,43}$ is apparent.

'M. S. Roos, R. E. Apfel, and S. C. Wardlaw, "Application of 30-MHz acoustic scattering to the study of human red blood cells," J. Acoust. Soc. Am. 83, 1639-1644 (1988).

${ }^{2}$ L. R. Abts, R. T. Beyer, P. D. Richardson, P. M. Galletti, and K. E. Karlson, "Microparticle detection with focused ultrasound," J. Acoust. Soc. Am. Suppl. 1 60, S53-S54 (1976).

${ }^{3}$ L. R. Abts, R. T. Beyer, P. D. Richardson, P. M. Galletti, and K. E. Karlson, "Reflections from microparticles in a flowing liquid," J. Acoust. Soc. Am. Suppl. 1 64, S62 (1978).

${ }^{4} \mathrm{P}$. L. Edwards and J. Jarzynski, "Scattering of focused ultrasound by spherical microparticles," J. Acoust. Soc. Am. 74, 1006-1012 (1983).

${ }^{5}$ M. S. Roos, "A technique for the study of acoustic scattering from microparticles,” J. Acoust. Soc. Am. 83, 770-776 (1988).

6J. Y. Chapelon, P. M. Shankar, and V. L. Newhouse, "Ultrasonic measurement of bubble cloud size profiles," J. Acoust. Soc. Am. 78, 196-201 (1985).

${ }^{7}$ S. T. Forbes and O. Nakken, Eds., "Manual of methods for fisheries resource survey and appraisal. Part 2. The use of acoustic instruments for fish detection and abundance estimation," FAO Man. Fish. Sci. 5, 1-138 (1972).

${ }^{8}$ M. L. Peterson, C. S. Clay, and S. B. Brandt, "Acoustic estimates of fish density and scattering function," J. Acoust. Soc. Am. 60, 618-622 (1976).

${ }^{9} J$. E. Ehrenberg, "A review of in situ target strength estimation techniques," FAO Fish. Rep. 300, 85-90 (1983).

${ }^{10}$ D. V. Holliday, "Extracting bio-physical information from the acoustic signatures of marine organisms," in Oceanic Sound Scattering Prediction, edited by N. R. Andersen and B. J. Zahuranec (Plenum, New York, 1977), pp. 619-624.

"R. E. Pieper, "Euphausiid distribution and biomass determined acoustically at 102 kHz," Deep-Sea Res. 26, 687-702 (1979).

${ }^{12}$ T. K. Stanton, R. D. M. Nash, R. L. Eastwood, and R. W. Nero, “A field examination of acoustical scattering from marine organisms at $70 \mathrm{kHz}$," IEEE J. Ocean. Eng. OE-12, 339-348 (1987).

${ }^{13}$ D. V. Holliday, R. E. Pieper, and G. S. Kleppel, "Determination of zooplankton size and distribution with multifrequency acoustic technology," J. Cons. Int. Explor. Mer 46, 52-61 (1989).

${ }^{14} J$. R. Proni, F. C. Newman, D. C. Rona, D. E. Drake, G. A. Berberian, C. A. Lauter, Jr., and R. L. Sellers, "On the use of acoustics for studying suspended oceanic sediment and for determining the onset of the shallow thermocline," Deep-Sea Res. 23, 831-837 (1976).

${ }^{15}$ M. H. Orr and R. R. Hess, "Remote acoustic monitoring of natural suspensate distributions, active suspensate resuspension, and slope/shelf water intrusions," J. Geophys. Res. 83(C8), 4062-4068 (1978).

${ }^{16} \mathrm{~A}$. E. Hay, "On the remote acoustic detection of suspended sediment at long wavelengths," J. Geophys. Res. 88(C12), 7525-7542 (1983). 
${ }^{17} J$. Sheng and A. E. Hay, "An examination of the spherical scatterer approximation in aqueous suspensions of sand," J. Acoust. Soc. Am. 83, 598-610 (1988).

${ }^{18} \mathrm{E}$. Ona, "The equivalent beam angle and its effective value when applying an integrator threshold," Counc. Meet. Int. Counc. Explor. Sea 1987/ B:35, Copenhagen, Denmark.

${ }^{19}$ D. H. Cushing, "Computations with a sonar equation," J. Cons. Int. Explor. Mer 35, 22-26 (1973).

${ }^{20}$ D. H. Cushing, "The present state of acoustic survey," J. Cons. Int. Explor. Mer 38, 28-32 (1978).

${ }^{21} \mathrm{~K}$. I. Yudanov and I. L. Kalikhman, "Sound scattering by marine animals," in Proceedings of the Meeting on Hydroacoustical Methods. for the Estimation of Marine Fish Populations, edited by J. B. Suomala, Jr. (C. S. Draper Lab., Cambridge, MA, 1981), Vol. 2, pp. 53-95.

${ }^{22}$ I. L. Kalikhman, W. D. Tesler, and K. I. Yudanov, "Methods of determining the density of fish concentrations," in Proceedings of the Meeting on Hydroacoustical Methods for the Estimation of Marine Fish Populations, edited by J. B. Suomala, Jr. (C. S. Draper Lab., Cambridge, MA, 1981), Vol. 2, pp. 533-573.

${ }^{23}$ A. Aglen, "Echo integrator threshold and fish density distribution," FAO Fish. Rep. 300, 35-44 (1983).

${ }^{24} I$. L. Kalikhman and W. D. Tesler, "The effective parameters of the real acoustic beam," FAO Fish. Rep. 300, 9-17 (1983).

${ }^{25}$ H. Lassen, "Signal threshold in echointegration," Counc. Meet. Int. Counc. Explor. Sea 1986/B:35, Copenhagen, Denmark.

${ }^{26}$ R. T. Weimer and J. E. Ehrenberg, "Analysis of threshold-induced bias inherent in acoustic scattering cross-section estimates of individual fish," J. Fish. Res. Board Can. 32, 2547-2551 (1975).

${ }^{27}$ C. S. Clay and H. Medwin, Acoustical Oceanography: Principles and Applications (Wiley, New York, 1977).

${ }^{28}$ D. N. MacLennan, "Acoustical measurement of fish abundance," J. Acoust. Soc. Am. 87, 1-15 (1990).

${ }^{29} \mathrm{~K}$. G. Foote, "Correcting acoustic measurements of scatterer density for extinction," J. Acoust. Soc. Am. 88, 1543-1546 (1990).

${ }^{30} \mathrm{~K}$. G. Foote, "Biasing of fish abundance estimates derived from use of the sector scanning sonar in the vertical plane," in Proceedings of the Confer- ence Progress in Sector Scanning Sonar (Institute of Acoustics, Edinburgh, 1979), pp. 44-52.

${ }^{31}$ E. J. Simmonds, "A comparison between measured and theoretical equivalent beam angles for seven similar transducers," J. Sound Vib. 97, 117128 (1984).

${ }^{32} \mathrm{~K}$. V. Mackenzie, "Nine-term equation for sound speed in the oceans," J. Acoust. Soc. Am. 70, 807-812 (1981).

${ }^{33}$ R. E. Francois and G. R. Garrison, "Sound absorption based on ocean measurements. Part II: Boric acid contribution and equation for total absorption," J. Acoust. Soc. Am. 72, 1879-1890 (1982).

${ }^{34} \mathrm{O}$. Nakken and K. Olsen, "Target strength measurements of fish," Rapp. P.-v. Réun. Cons. Int. Explor. Mer 170, 52-69 (1977).

${ }^{35}$ K. G. Foote and $O$. Nakken, "Dorsal aspect target strength functions of six fishes at two ultrasonic frequencies," Fisken og havet, Ser. B 1978(3), 1-95.

${ }^{36}$ R. J. Urick, Principles of Underwater Sound (McGraw-Hill, New York, 1975), 2nd ed.

${ }^{37}$ K. G. Foote and E. Ona, "Tilt angles of schooling penned saithe," J. Cons. Int. Explor. Mer 43, 118-121 (1987).

${ }^{38} \mathrm{~K}$. Olsen, "Orientation measurements of cod in Lofoten obtained from underwater photography and their relation to target strength," Counc. Meet. Int. Counc. Explor. Sea 1971/B:17, Copenhagen, Denmark.

${ }^{39}$ K. G. Foote, “Acoustic sampling volume for cod," Counc. Meet. Int. Counc. Explor. Sea 1989/B:5, Copenhagen, Denmark.

${ }^{40}$ E. D. Hirleman, V. Oechsle, and N. A. Chigier, "Response characteristics of laser diffraction particle size analyzers: optical sample volume extent and lens effects," Opt. Eng. 23, 610-619 (1984).

${ }^{41}$ B. A. Weiss, P. Derov, D. DeBiase, and H. C. Simmons, "Fluid particle sizing using a fully automated optical imaging system," Opt. Eng. 23, 561-566 (1984).

${ }^{42} \mathrm{H}$. Nakane and Y. Sasano, "Structure of a sea-breeze front revealed by scanning lidar observation,” J. Meteoral. Soc. Jpn. 64, 787-792 (1986).

${ }^{43}$ P. Qing, H. Nakane, Y. Sasano, and S. Kitamura, "Numerical simulation of the retrieval of aerosol size distribution from multiwavelength laser radar measurements," Appl. Opt. 28, 5259-5265 (1989). 